\title{
Evidence of natural occurrence of the banned antibiotic chloramphenicol in herbs and grass
}

\author{
Bjorn Berendsen • Linda Stolker • Jacob de Jong • Michel Nielen • \\ Enkhtuya Tserendorj • Ruuragchas Sodnomdarjaa • Andrew Cannavan • \\ Christopher Elliott
}

Received: 24 February 2010 /Revised: 2 April 2010 / Accepted: 3 April 2010 /Published online: 30 April 2010

(C) The Author(s) 2010. This article is published with open access at Springerlink.com

\begin{abstract}
Chloramphenicol (CAP), a broad-spectrum antibiotic, was detected in several herb and grass samples from different geographic origins. Due to its suspected carcinogenicity and linkages with the development of aplastic anemia in humans, CAP is banned for use in foodproducing animals in the European Union (EU) and many other countries. However, products of animal origin originating from Asian countries entering the European market are still found noncompliant (containing CAP) on a regular basis, even when there is no history of chloramphenicol use in these countries. A possible explanation for the continued detection of these residues is the natural occurrence of CAP in plant material which is used as
\end{abstract}

B. Berendsen $\cdot$ L. Stolker $(\bowtie) \cdot J$. de Jong $\cdot$ M. Nielen

RIKILT, Wageningen UR,

Akkermaalsbos 2, P.O. Box 230,

6700 AE Wageningen, The Netherlands

e-mail: linda.stolker@wur.nl

M. Nielen

Laboratory of Organic Chemistry, Wageningen University,

Dreijenplein 8 ,

6703 HB Wageningen, The Netherlands

E. Tserendorj $\cdot$ R. Sodnomdarjaa

Ministry of Food and Agriculture,

Veterinary Diagnostic Laboratory,

P.O. Box 53-03, Ulaanbaatar 210153, Mongolia

A. Cannavan

FAO/IAEA Agriculture and Biotechnology Laboratory,

2444 Seibersdorf, Austria

C. Elliott

Institute of Agri-Food and Land Use,

School of Biological Sciences, Queens University Belfast,

Stranmillis Road,

Belfast BT9 5AG, UK animal feed, with the consequent transfer of the substance to the animal tissues. Approximately 110 samples were analyzed using liquid chromatography coupled with mass spectrometric detection. In 26 samples, the presence of CAP was confirmed using the criteria for banned substances defined by the EU. Among other plant materials, samples of the Artemisia family retrieved from Mongolia and from Utah, USA, and a therapeutic herb mixture obtained from local stores in the Netherlands proved to contain CAP at levels ranging from 0.1 to $450 \mu \mathrm{g} / \mathrm{kg}$. These findings may have a major impact in relation to international trade and safety to the consumer. The results of this study demonstrate that noncompliant findings in animalderived food products may in part be due to the natural occurrence of chloramphenicol in plant material. This has implications for the application of current EU, USA, and other legislation and the interpretation of analytical results with respect to the consideration of CAP as a xenobiotic veterinary drug residue and the regulatory actions taken upon its detection in food.

Keywords Plant material · 2002/657/EC · Confirmation . Analysis $\cdot$ LC-MS/MS $\cdot$ Chloramphenicol

\section{Introduction}

Chloramphenicol (CAP) is a broad-spectrum antibiotic with historical veterinary uses in all major food-producing animals. CAP is biosynthesized by the soil organism Streptomyces venezuelae and several other actinomycetes but is produced for commercial use by chemical synthesis [1]. The drug has been evaluated by a number of agencies, including the International Agency for Research on Cancer (1990), the European Committee for Veterinary Medicinal 
Products (1994), the US Food and Drug Administration (1985), and more recently in 2005 the Joint Expert Committee on Food Additives (JECFA, FAO) at its 62nd meeting [2]. CAP is a suspected carcinogen, and for this reason the drug is banned for use in food-producing animals in the European Union (EU) and in many other countries, including the USA, Canada, Australia, Japan, and China. A series of EU decisions describe the required testing for animal-derived food products entering the European market [3-5]. A minimum required performance limit (MRPL) of $0.3 \mu \mathrm{g} / \mathrm{kg}$ was assigned by the European Commission for the analytical methods testing for CAP in products of animal origin [6]. Furthermore, the MRPL is the reference point for action in relation to the evaluation of consignments of food. In recent years, findings of CAP residues in food products such as poultry, honey, and sheep casings have had a major impact on international trade [7]. Follow-up investigations in Asian countries that were related to the noncompliant findings could not identify the origin of CAP residues and found no recent history of CAP use.

Various hypotheses have been suggested to explain these results. Residues may be caused by the illegal use of the drug in animal production, through contamination of the products by processing workers who were using topical human medicines containing CAP, or by ingestion of naturally occurring CAP from the environment. Due to the fact that recent findings of CAP in several products produced in different countries, such as Thailand and Mongolia, could not be explained by the use of the drug, the hypothesis of naturally occurring CAP warranted scientific investigation.

Several hypotheses for the contamination of food products by possible naturally occurring CAP are described by the JECFA. The possibility of contamination due to ingestion of naturally or externally contaminated soil was evaluated. The final conclusion from the evaluation was that the committee could not completely rule out the possibility that foods are occasionally contaminated from environmental sources. However, due to lack of analytical methods to detect the relevant concentrations of CAP in soil, there are no analytical data available to support this suggestion.

Another hypothesis, which to our knowledge has never been investigated, is the possibility that grass and herbs (plant materials) absorb and accumulate CAP from the soil. The CAP-containing grass and herbs are used as pasture or harvested as animal feed or forage, and consequently products of animal origin are contaminated with residues of CAP. It has been shown that plants are able to absorb veterinary drugs such as tetracyclines from soil [8]. To test this hypothesis, several samples of grass and herbs were collected from Mongolia where the contamination of food products with CAP has been identified previously. Samples of grass and of herbs belonging to the Artemisia and Thalictrum families were collected. These herbs were selected for collection because it is known that these plants have a bitter taste (as does CAP) and are used as traditional medicines by the local population. To determine if CAP presence could be detected in herbs grown at other locations, samples were purchased from a number of retail outlets in the Netherlands.

For the detection, quantification, and confirmation of CAP, different analytical methods are available based on both gas chromatography and liquid chromatography combined with mass spectrometry (GC-MS or LC-MS) [9-12]. For monitoring purposes, the most frequently used technique is the highly selective, sensitive, and relatively quick LC combined with tandem mass spectrometric (MS/ MS) detection. This technique is able to detect CAP at the MRPL level of $0.3 \mu \mathrm{g} / \mathrm{kg}$ in various food products.

In the present study, plant material and therapeutic herb mixtures were analyzed for the presence of CAP using an LC-MS/MS method which was validated in compliance with the EU guidelines in Commission Decision 2002/657/ $\mathrm{EC}$ and accredited in compliance with NEN-EN-ISO/IEC 17025. However, very recently, Schürmann et al. [13] demonstrated that for a specific matrix/analyte combination a false noncompliant result is obtained by using the EU identification points approach. Therefore, for additional selectivity, a few representative samples were reanalyzed using a highly selective very high-pressure LC system (VHPLC) monitoring three selected reaction monitoring (SRM) transitions.

The major aim of the present study was to determine if CAP can occur naturally in herbs and grass. If confirmed, this observation would help to explain the noncompliant findings of CAP in products of animal origin even when there is no recent history of CAP use. Animals grazing on pasture where such herbaceous plants are prevalent or being fed with feedstuffs containing those plants may become contaminated with CAP, with the subsequent detection of CAP in the animal products.

\section{Materials}

Chemicals, reagents, and solutions

Methanol (HPLC supra-gradient grade), dichloromethane, ammonia (25\%), and toluene were obtained from Biosolve (Valkenswaard, the Netherlands). CAP (Sigma-Aldrich, St. Louis, MO, USA) and ${ }^{37} \mathrm{Cl}_{2}$-CAP (RIVM, Bilthoven, the Netherlands) were used as reference standards. The stock solution of the CAP reference standard was prepared in methanol at $100 \mu \mathrm{g} / \mathrm{l}$ and was stored at $-18^{\circ} \mathrm{C}$. Dilutions of 
these stock solution were all prepared in Milli-Q water and stored at $4{ }^{\circ} \mathrm{C}$. The stability of CAP stock solution at $4{ }^{\circ} \mathrm{C}$ is at least 6 months.

A solution of ammonia $(0.025 \%)$ was prepared by diluting $1 \mathrm{ml}$ ammonia (25\%) in 11 of Milli-Q water.

\section{Samples}

Fifteen plant material samples, among which were Artemisia frigida and Thalictrum simplex, were collected from local fields in the neighborhood of the State Central Veterinary Laboratory, Mongolia (Atar province, autumn 2007). The first set of five samples was transported in May 2008 to the EU laboratory. A second set of ten samples arrived in June of the same year. Six therapeutic herb mixtures, including teas, claiming an anti-infectious effect, were obtained from a local store in the Netherlands (June 2009). One A. frigida sample originating from Utah, USA, was obtained by Internet order from a retail outlet in the UK (June 2009).

In September 2009, samples of herb (Artemisia sieversiana, A. frigida, and green grass) were collected from five different areas in Mongolia (Lun, Atar, Hui doloon hudag, Erdene, and Bayandelger). In each area, three different locations were selected, and at each location three samples of herbs were collected. Each sample of herb was split into leaves, roots, and, if available, stalk. Furthermore, together with each sample of herb, two samples of soil were collected (directly below the surface and $20 \mathrm{~cm}$ below the surface). Finally, a total of five samples of water were collected. The total number of samples collected was 192 .

\section{Liquid chromatography}

The separation of CAP from the sample components was carried out using LC or by VHPLC.

The LC system consists of a vacuum degasser, autosampler, and a binary pump (Acquity Waters, Milford, MA, USA) equipped - for LC applications - with an X-Bridge $\mathrm{C}_{18}$ analytical column, $3.0 \times 15 \mathrm{~mm}, 5 \mu \mathrm{m}$ (Waters), placed in a column oven at $30{ }^{\circ} \mathrm{C}$. Isocratic elution was performed with a mobile phase of ammonia( $0.025 \%)$-acetonitrile $(45: 55, v / v)$ at a flow rate of $0.4 \mathrm{ml} / \mathrm{min}$. Injection volume was $100 \mu 1$.

For VHPLC applications, the LC was equipped with a Waters Aqcuity UPLC BEH $\mathrm{C}_{18}$ analytical column of $2.1 \times$ $50 \mathrm{~mm}, 1.7 \mu \mathrm{m}$ (Waters) placed in a column oven at $50{ }^{\circ} \mathrm{C}$. The gradient (solvent $\mathrm{A}$, water $(100 \%)$; solvent $\mathrm{B}$, methanol $(100 \%)$ ) was $0-0.5 \mathrm{~min}, 10 \% \mathrm{~B}, 0.5-3.5 \mathrm{~min}$, linear increase to $100 \%$ B with a final hold of 0.5 min. Under these conditions, CAP eluted after $2.7 \mathrm{~min}$. Injection volume was $100 \mu 1$.
Mass spectrometry

Detection was carried out using a Waters Quattro Ultima mass spectrometer with electrospray ionization operating in negative ionization mode. The operating parameters were: capillary voltage, $2.7 \mathrm{kV}$; cone voltage, $25 \mathrm{~V}$; source temperature, $120{ }^{\circ} \mathrm{C}$; desolvation temperature, $300{ }^{\circ} \mathrm{C}$; cone gas flow, $2001 \mathrm{~h} \mathrm{~h}^{-1}$; and desolvation gas, $500 \mathrm{l} \mathrm{h}^{-1}$. CAP was fragmented using collision-induced dissociation, and SRM transitions at $m / z=321.0>152.1$ and $m / z=321.0>194.0$ were monitored. In the VHPLC-MS/MS, an additional transition was monitored: $m / z=321.0>257.1 .{ }^{37} \mathrm{Cl}_{2}$-CAP was detected by monitoring the transition $m / z=324.8>152.0$. Data were acquired and processed using MassLynx 4.1 software (Waters).

\section{Methods}

Analytical method

Plant material was cut into small pieces and pulverized using a Moulinex blender.

Small pieces of plant sample material $(1 \mathrm{~g})$ or soil $(2 \mathrm{~g})$ were weighed into a 50-ml tube, and internal standard ${ }^{37} \mathrm{Cl}_{2}$ CAP was added. For the quality control (QC) samples, CAP reference standard solution was also added. Next, $10 \mathrm{ml}$ of Milli-Q water (or more, with a maximum of $25 \mathrm{ml}$ in cases where the water was completely absorbed by the sample material) was added to the sample, and CAP was extracted from the material by shaking (rotary tumbler, $10 \mathrm{~min}$ ) after which it was centrifuged $(15 \mathrm{~min}, 3,500 \times \mathrm{g})$. An aliquot $(3 \mathrm{ml})$ of the extract was transferred to an Extrelut ${ }^{\circledR}$ NT3 (Merck, Darmstadt, Germany) column. After at least 20-min equilibration, CAP was extracted from the cartridge using $15 \mathrm{ml}$ dichloromethane which was collected in a $12-\mathrm{mm}$ polypropylene centrifuge tube. The dichloromethane was evaporated to dryness under a stream of nitrogen at $35^{\circ} \mathrm{C}$, and the residue was dissolved in $0.5 \mathrm{ml}$ Milli-Q water. The final extract was shaken with $1 \mathrm{ml}$ toluene after which the aqueous layer was transferred into an LC vial.

\section{Quantification}

For quantification, a "detector response" - peak area ratios IS/Standard CAP_-versus "CAP concentration" plot was constructed. To this end, blank - screened "negative" for CAP in previous research-plant (or soil) samples were fortified with different concentrations of CAP $(0-50 \mu \mathrm{g} / \mathrm{kg})$ and used as matrix-matched standards (MMS-s). The collected samples were analyzed together with the MMS$\mathrm{s}$; concentrations were calculated using the least squares linear regression method. 


\section{Identification and confirmation}

According to the EU criteria [14], the identity of CAP is considered to be confirmed when a minimum of four identification points is earned. LC-MS/MS monitoring two SRM transitions and comparing the SRM ion ratio from sample and standard is a suitable technique to obtain the requested number of identification points. Furthermore, the following criteria have to be applied:

- The relative retention time of the compound in the sample has to be the same as the relative retention time of the reference within a margin of $2.5 \%$.

- The ion ratio of two SRM transition ions of the compound in the sample has to be within a specific tolerance interval around the ion ratio of the reference (for example, interval of $20 \%$ if the ion ratio is above $50 \%$ and an interval of $25 \%$ if the ion ratio is between $20 \%$ and $50 \%$ ).

\section{Validation}

The LC-MS/MS method used for the determination and identification of CAP was validated according to guidelines described for quantitative confirmatory methods in Commission Decision 2002/657/EC [14]. Previous full validation was performed for the matrices urine and shrimps at the concentration levels of $0.2,0.4$, and $0.6 \mu \mathrm{g} / \mathrm{kg}(0.5$ through two times the MRPL). All method characteristics, including linearity, repeatability, and reproducibility, fulfilled the EU criteria. The $\mathrm{CC} \alpha$ an $\mathrm{CC} \beta$, established by the analysis of 20 blank samples and 20 fortified samples, were, respectively, 0.05 and $0.2 \mu \mathrm{g} / \mathrm{kg}$ for urine and 0.05 and $0.15 \mu \mathrm{g} / \mathrm{kg}$ for shrimps. Additional validation experiments were performed for the matrices milk, animal feed, and plant material including leaves, stalk, roots, and soil. The additional 1-day validation for plant material was carried out at levels of 0.3 and $0.5 \mu \mathrm{g} / \mathrm{kg}$ ( $n=6$ at each level). From these experiments, the repeatability was established and compared with the results obtained for the matrices urine and shrimps. In case there are no significant differences, the $\mathrm{CC} \alpha$ for the additional matrices is established based on the results obtained during the initial validation study.

\section{Sample analysis}

The samples of plant material and soil were analyzed in a series with a maximum of 40 samples. Each series of samples started and ended with the analysis of matrix-matched calibration standards. The samples were analyzed by the method and the experimental conditions as described above.
Furthermore, for confirmation of the identity of CAP, the criteria for confirmation ("Identification and confirmation") had to be fulfilled.

For additional selectivity and confirmation purposes, the CAP-containing samples (confirmed by using LC) and some blank samples were reanalyzed by VHPLC. As a reference, the average relative retention time and SRM ion ratios of blank plant material samples spiked with CAP at 3.0 and $10.0 \mu \mathrm{g} / \mathrm{kg}$ were used. Three SRM transitions were monitored, and the ion ratios of the sample and the spiked samples were compared. In case the ion ratios fulfill the EU criteria (ion ratio of sample within the tolerance interval of the spiked samples), the identity of CAP was unambiguous.

\section{Results and discussion}

\section{Validation}

The accuracy obtained for the analyses of six samples of plant material (leaves, roots + soil, stalk) at levels of 0.3 and $0.5 \mu \mathrm{g} / \mathrm{kg}$ ( $n=6$ at each level) was, respectively, $100 \%$ and $104 \%$, and the relative standard deviation under repeatability conditions was $9 \%$ and $6 \%$ at these levels. These results did not significantly differ from the results obtained in the initial validation. Based on these results, it was concluded that the $\mathrm{CC} \alpha$ for plant material is $<0.1 \mu \mathrm{g} / \mathrm{kg}$ (second lowest point of the calibration curve). In other words, the method is suitable to detect CAP in plant material at concentrations levels $\geq 0.1 \mu \mathrm{g} / \mathrm{kg}$.

In all cases, the quantification was carried out using a matrix-matched calibration curve in the concentration range of 0.05 to $2 \mu \mathrm{g} / \mathrm{kg}$.

Sample analysis

In August 2009, the first set of 22 samples including: (a) the samples collected in Mongolia during 2007; (b) the samples obtained from a local store in the Netherland; and (c) the A. frigida obtained from Utah, USA, were analyzed for CAP content by using LC-MS/MS. The data produced have been presented in Table 1. It can be seen that, in all the herb samples from Mongolia, CAP was detected at concentrations up to $450 \mu \mathrm{g} / \mathrm{kg}$. Furthermore, in one herb mixture obtained from a local store in the Netherlands and the sample of $A$. frigida obtained from a retail outlet, CAP was detected at low microgram per kilogram levels.

If the concentrations $(0.3-3 \mu \mathrm{g} / \mathrm{kg})$ of CAP detected in products of animal origin during monitoring in the EU are compared to these findings (EU Rapid Alert System for Food and Feed 2008), then the possibility of the sources of some of this CAP originating from plant materials must be considered a possibility. 
Table 1 Concentrations of chloramphenicol detected in herb(mixture) by LC-MS/MS

\begin{tabular}{|c|c|c|c|c|}
\hline Description & Code & Sample name & Type of plant material & Result $(\mu \mathrm{g} / \mathrm{kg})$ \\
\hline \multirow[t]{5}{*}{ First set Mongolian plants (collected autumn 2007) } & $S 1$ & Thalictrum simplex & Herb & 23 \\
\hline & $S 2$ & Artemisia sieversiana & Herb & 46 \\
\hline & $S 3$ & Artemisia frigida & Herb & 175 \\
\hline & $S 4$ & Thermopsis daurica & Herb & 21 \\
\hline & S5 & Thalictrum simplex & Herb & 0.3 \\
\hline \multirow[t]{10}{*}{ Second set Mongolian plants (collected autumn 2007) } & S6 & Artemisia sieversiana & Herb & 160 \\
\hline & $S 7$ & Thermopsis daurica & Herb & 25 \\
\hline & $S 8$ & Artemisia sieversiana & Herb & 20 \\
\hline & S9 & Thalictrum simplex & Herb & 40 \\
\hline & $S 10$ & Thalictrum simplex & Herb & 450 \\
\hline & $S 11$ & Thalictrum simplex & Herb & 15 \\
\hline & $S 12$ & Artemisia sieversiana & Herb & 8 \\
\hline & $S 13$ & Thalictrum simplex & Herb & 50 \\
\hline & $S 14$ & Artemisia sieversiana & Herb & 4 \\
\hline & $S 15$ & Artemisia sieversiana & Herb & 5 \\
\hline Utah, $\mathrm{USA}^{\mathrm{a}}$ & & Artemisia frigida & Herb & 1.3 \\
\hline & & Kamillebloesem & Herb & ${ }^{\mathrm{b}}$ \\
\hline & & Bandrek 2 pigeons & Herb tea & $-{ }^{\mathrm{b}}$ \\
\hline & & Parusahaan Jamu, kruiden & Herb mixture & 4 \\
\hline & & Ge Xian Weng & Herb tea & $-{ }^{\mathrm{b}}$ \\
\hline & & Giju & Herb tea & $-{ }^{\mathrm{b}}$ \\
\hline & & Kruidenmix & Herb mixture & $-{ }^{\mathrm{b}}$ \\
\hline & & Echinacea force & Herb medicine & $-{ }^{\mathrm{b}}$ \\
\hline
\end{tabular}

${ }^{\text {a }}$ Obtained through a retail outlet in the UK (Internet order)

${ }^{\mathrm{b}}<0.1 \mu \mathrm{g} / \mathrm{kg}$

The data generated also show that the accumulation of CAP in plants does not occur at all locations and at all times. As a follow-up investigation into the variability of CAP concentrations found in the first survey, in September
2009, some additional samples were collected from Mongolian pastures. In total, 192 samples of leaves, roots, stalk of $A$. sieversiana and A. frigida were collected as well as samples of green grass, soil, and water. From these 192
Table 2 Concentrations of chloramphenicol detected in Mongolian herb samples collected September 2009

\begin{tabular}{lcllc}
\hline Area & Location/sample no. & Name & Part of the plant & Result $(\mu \mathrm{g} / \mathrm{kg})$ \\
\hline Lun & $9 / 36$ & Green grass & Leaves & 0.6 \\
& $9 / 37$ & Green grass & Roots & 0.3 \\
Atar & $14 / 59$ & A. frigida & Roots & 0.3 \\
& 1563 & Green grass & Roots & 0.6 \\
& $16 / 66$ & A. sieversiana & Leaves & 2.8 \\
& $17 / 72$ & A. frigida & Roots & 3.8 \\
Hui doloon hudag & $19 / 80$ & A. sieversiana & Roots & 2.0 \\
& $21 / 86$ & Green grass & Leaves & 1.2 \\
& $24 / 100$ & Soil & & 0.1 \\
& $25 / 103$ & A. frigida & Roots & 3.0 \\
Bayandelger & $39 / 168$ & A. frigida & Leaves & 0.3 \\
& $43 / 185$ & Green grass & Roots & 0.3 \\
& $43 / 186$ & Soil & Up & 0.2 \\
\hline
\end{tabular}

${ }^{a}$ Soil samples were taken direct under the surface (=up) and in the plant hole (=below) 
Fig. 1 Results of the analysis of $\mathrm{CAP}$ in $\mu \mathrm{g} / \mathrm{kg}$ for the subset of 87 samples collected in Mongolia (Autumn 2009) per area

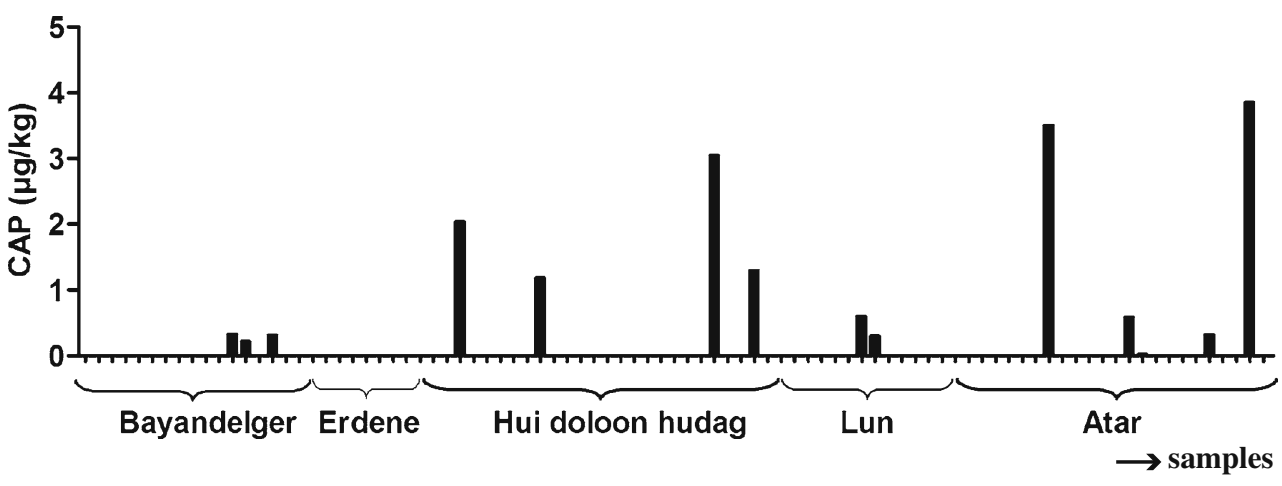

samples, a representative set of 87 samples were analyzed for CAP. Table 2 presents the results for samples containing $\mathrm{CAP} \geq 0.1 \mu \mathrm{g} / \mathrm{kg}$.

From the results in Table 2, it can be observed that only a small selection of the samples, 13 out of $87(=15 \%)$, contain CAP at detectable concentrations. From these 13 samples, only five samples contain CAP between 1 and $5 \mu \mathrm{g} / \mathrm{kg}$. All other concentrations found in the samples were $<1 \mu \mathrm{g} / \mathrm{kg}$. Furthermore, no specific relationship was found between the concentration of CAP in soil and herbs and concentrations found at a specific location. The samples containing CAP appear to be randomly distributed across the population of samples. This finding is further demonstrated in Figs. 1, 2, and 3.

Figure 1 presents the results of the subset of 87 samples per province. All areas have CAP-containing plants except one (Atar area). Figure 2 illustrates that CAP was found in all three species of plants tested, but not in every sample. Figure 3 points towards the plant roots as having the highest concentration of CAP compared to leaves and soil samples. From these data, it was concluded that the herbs growing on the Mongolian pastures do not always contain high concentrations of CAP and also that no single herb family appears to be responsible for the bioaccumulation of the antimicrobial compound.

It is difficult to draw general conclusions from the observations made thus far, but it is clear that a relatively large number of root samples contain CAP. It is therefore hypothesized that the CAP originates from the soil and is absorbed through the plant roots, regardless of plant type. The soil organism $S$. venezuelae, and some actinomycetes, are known to produce CAP, but biosynthesis depends on many factors including the external conditions in the soil. Consequently, it is proposed that the production of CAP may depend on environmental conditions such as the prevailing temperature and the amount of rainfall and consequent moisture content of the soil. The year 2007, for example, was very dry for Mongolia whereas the year 2009 was a very wet year. It is possible (though not proven by our experiments) that the differences in climatic conditions have a strong influence on the biosynthesis of CAP by microorganisms in the soil and its absorption by the plants' root system and therefore on the concentration of CAP in the plants. Further research is necessary to confirm the biosynthesis of CAP by soil microorganisms in the vicinity of plants found to contain CAP and to elaborate the various factors influencing CAP biosynthesis and uptake by plants.

\section{Confirmation}

Nowadays, the unambiguous identification of a prohibited compound is of high importance due to the financial consequences of a (false) noncompliant finding, which
Fig. 2 Results of the analysis of CAP for the subset of 87 samples collected in Mongolia (autumn 2009) per type of plant

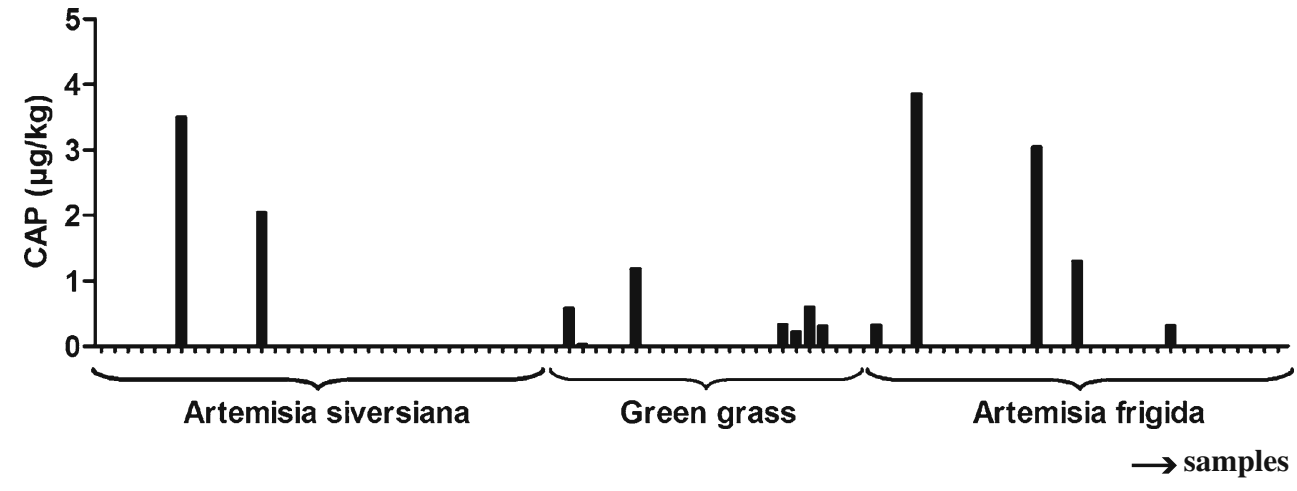


Fig. 3 Results of the analysis of CAP for the subset of 87 samples collected in Mongolia (autumn 2009) per sample material

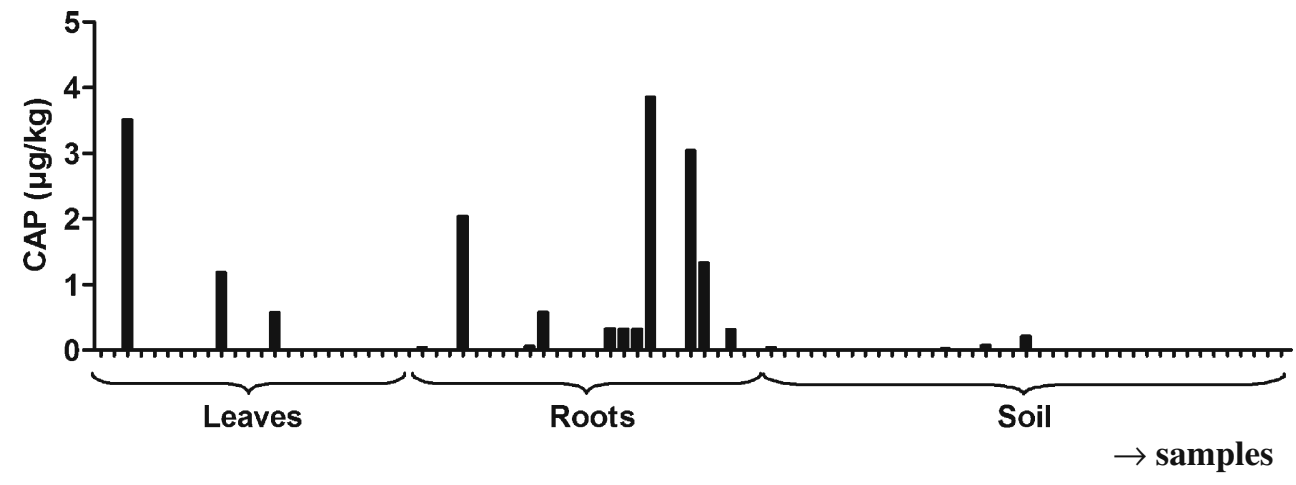

may include rejection of consignments of contaminated food products by the importing country, increased testing requirements at the expense of the exporter, and possibly prosecution and financial penalties for producers found to be illegally using the compound in animal production. Therefore, additional precautions are necessary for the identification of CAP in plant materials because of the possible impact the results could have for the international market.

For unambiguous identification of a prohibited (banned) compound, the EU criteria described in "Identification and confirmation" have to be fulfilled. The EU criteria are set up for products of animal origin, but these criteria were also used in this study to confirm the identity of CAP in plant material.

The results of two representative noncompliant (CAPcontaining) samples analyzed using LC-MS/MS are presented in Table 3. From the control samples, an average ion ratio of $39.5 \%$ and a relative retention time (RRT) of 1.008 is calculated. For confirmatory analysis according to EU criteria [14], the maximum allowed relative deviation of the ion ratio is $25 \%$ and, thus, in this case the identity of CAP is confirmed if the ion ratio is between $29.5 \%$ and $49.1 \%$. The maximum allowed deviation for the relative retention time is $2.5 \%$. The ion ratios obtained for the samples only slightly deviate from the reference ion ratio (maximum relative difference is $-2.5 \%$ ), and the relative retention time is 1.008 for all samples. From this, it is concluded that the identity of CAP is confirmed.
For additional selectivity and to obtain additional proof for confirmation, the samples were injected onto an VHPLC-MS/MS system to obtain a higher chromatographic resolution. Furthermore, three transitions were monitored, resulting in a total of 5.5 identification points demonstrating the high selectivity of this method.

Chromatograms of a blank herb mixture sample, a blank herb mixture sample fortified with $2 \mu \mathrm{g} / \mathrm{kg}$ CAP, a noncompliant herb mixture sample $(4 \mu \mathrm{g} / \mathrm{kg})$, and the same herb mixture sample with the addition of $2 \mu \mathrm{g} / \mathrm{kg}$ are presented in Fig. 4.

The results of representative noncompliant samples are presented in Table 4. From the control samples, an average ion ratio of $37.6 \%$ is calculated for the product ions $m / z=194$ versus 152 and $70.2 \%$ for $m / z=257$ versus 152 . Furthermore, an RRT of 1.004 is calculated. The ion ratios obtained for the samples are all within the tolerance intervals defined by the EU and presented in Table 4, and the relative retention time is 1.004 for all samples. From this, it is concluded that the identity of CAP is unambiguous.

The LC results did not deviate from the VHPLC results. In other words, all samples containing CAP based on LC results were (re)confirmed by using the VHPLC.

\section{Conclusions}

The LC-MS/MS analysis of plant materials from different origins, including herb mixtures obtained at local stores,

Table 3 LC-MS/MS results of two samples including identification characteristics

\begin{tabular}{rcccccc}
\hline Description (conc. CAP) & $\begin{array}{l}\text { RRT } \\
(\mathrm{min})\end{array}$ & $\begin{array}{l}\text { Rel. deviation of } \\
\text { RRT (\%) }\end{array}$ & $\begin{array}{l}\text { Response SRM } \\
321>152\end{array}$ & $\begin{array}{l}\text { Response SRM } \\
321>194\end{array}$ & $\begin{array}{l}\text { Ion ratio } \\
(\%)\end{array}$ & $\begin{array}{l}\text { Rel. deviation of ion } \\
\text { ratio (\%) }\end{array}$ \\
\hline Reference (blank +2 & $\mu \mathrm{g} / \mathrm{kg})$ & 1.008 & & & & 39.5 \\
Artemisia frigida $(1.3 \mu \mathrm{g} / \mathrm{kg})$ & 1.008 & 0 & 3,736 & 1,445 & 38.7 & -2.0 \\
Herb mixture $(4 \mu \mathrm{g} / \mathrm{kg})$ & 1.008 & 0 & 82,603 & 31,611 & 38.3 \\
\hline
\end{tabular}

${ }^{\mathrm{a}}$ Response $\mathrm{SRM}=$ peak area

${ }^{\mathrm{b}}$ Max tolerance percent according to 2002/657/EC criteria $25 \%$ 
a
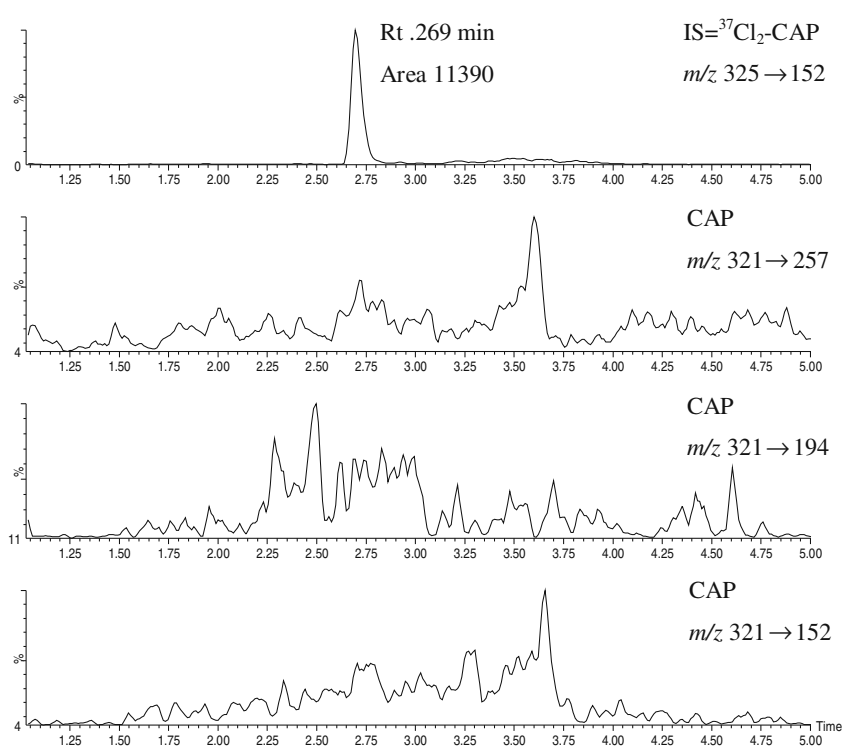

C
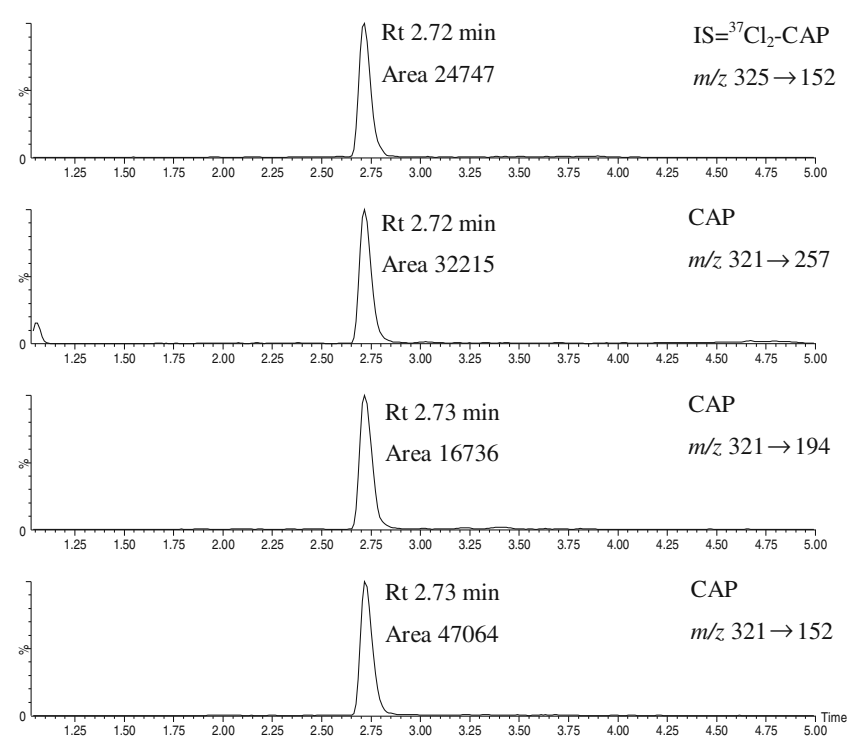

b

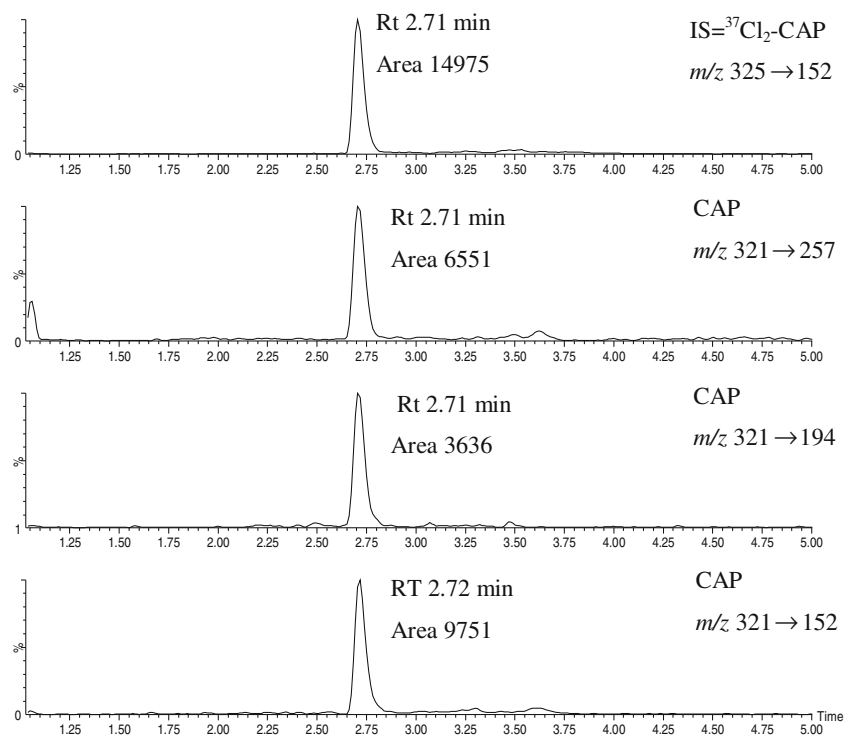

d
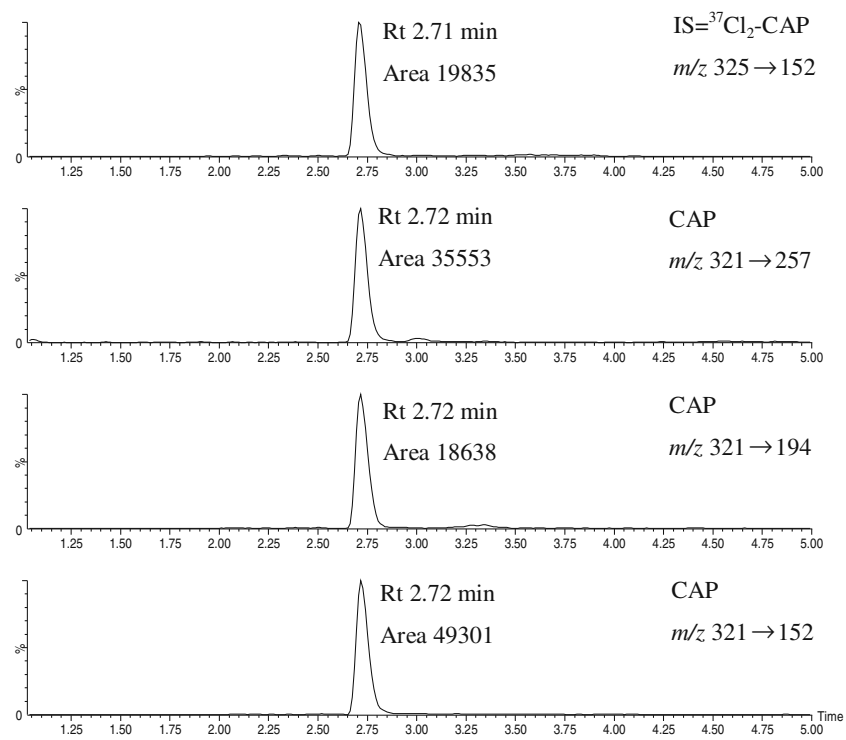

Fig. 4 VHPLC-MS/MS chromatograms showing three SRM transitions for CAP and one for the internal standard of a a blank herb sample, $\mathbf{b}$ a blank herb sample with addition of $2 \mu \mathrm{g} / \mathrm{kg}$ CAP, $\mathbf{c}$ a herb mixture from a local shop, and $\mathbf{d}$ sample $\mathbf{c}$ with addition of $2.0 \mu \mathrm{g} / \mathrm{kg} \mathrm{CAP}$

Table 4 HPLC-MS/MS results of three samples including identification characteristics

\begin{tabular}{|c|c|c|c|c|c|c|}
\hline Description (conc. CAP) & $\begin{array}{l}\text { RRT } \\
(\min )\end{array}$ & $\begin{array}{l}\text { Rel. dev. } \\
\text { Rt (\%) }\end{array}$ & $\begin{array}{l}\text { Ion ratio 194/ } \\
152(\%)\end{array}$ & $\begin{array}{l}\text { Rel. dev. ion ratio } \\
194 / 152(\%)^{\mathrm{a}}\end{array}$ & $\begin{array}{l}\text { Ion ratio } 257 / \\
152(\%)\end{array}$ & $\begin{array}{l}\text { Rel. dev. ion ratio } \\
257 / 152(\%)^{\mathrm{b}}\end{array}$ \\
\hline Reference (blank $+2 \mu \mathrm{g} / \mathrm{kg}$ ) & 1.004 & & 37.6 & & 70.2 & \\
\hline Artemisia F. $(1.3 \mu \mathrm{g} / \mathrm{kg})$ & 1.004 & 0 & 31.1 & -16.4 & 69.4 & -1.1 \\
\hline Artemisia F. $(175 \mu \mathrm{g} / \mathrm{kg})$ & 1.004 & 0 & 36.7 & -1.3 & 66.6 & -5.1 \\
\hline Herb mixture $(4 \mu \mathrm{g} / \mathrm{kg})$ & 1.004 & 0 & 35.6 & -4.3 & 68.5 & -2.4 \\
\hline
\end{tabular}

${ }^{\mathrm{a}}$ Max tolerance according to $2002 / 657 / \mathrm{EC}$ criteria $25 \%$

${ }^{\mathrm{b}}$ Max tolerance according to 2002/657/EC criteria $20 \%$ 
plant material obtained from Mongolian pastures, and a specific herb sample collected in Utah, USA, demonstrates that it is possible that plant materials can contain CAP. The concentrations of CAP varied from nondetectable up to $450 \mu \mathrm{g} / \mathrm{kg}$. In cases of noncompliant CAP results (concentrations above the $\mathrm{CC} \alpha$ of $0.1 \mu \mathrm{g} / \mathrm{kg}$ ), the identity of CAP was unambiguously confirmed according to EU criteria.

From the test results, it was concluded that plants belonging to different families can contain CAP. For example, CAP was detected in plants of the families Artemisia or Thalictrum, but it was also detected in grass. It is known that the soil organism $S$. venezuelae and related organisms can biosynthesize CAP. Therefore, it is suggested, based on the results obtained, that CAP is produced in the soil and that the plants absorb CAP through their root systems. Further research is required to confirm this supposition and to elaborate the environmental parameters affecting CAP occurrence in plants.

To the best of our knowledge, this is the first time that findings of CAP in plant materials has been reported. These findings make it a much more realistic prospect that products of animal origin can contain residues of CAP that are not due to (illegal) use of the drug, but rather due the natural occurrence of CAP. The results also have significant implications for the application of legislation with respect to the detection of CAP in food products and may imply, if not a change in the legislation, at least a change in the interpretation of analytical results and in follow-up actions and penalties to producers for the suspected illegal use of CAP.

Furthermore, the finding of CAP in samples of herbal products bought at retail outlet must be a cause for concern in relation to human exposure to this suspected carcinogen.

Acknowledgements This project was financially supported by the Dutch Ministry of Agriculture, Nature and Food Quality (project 7166601 and 7259301). Mrs. Enkhtuya Tserendorj was financially supported by the Joint FAO/IAEA Programme for nuclear techniques in Food and Agriculture. We thank Thijs Meijer and Hester Eusman for their technical assistants during the method validation and sample analysis.

Open Access This article is distributed under the terms of the Creative Commons Attribution Noncommercial License which permits any noncommercial use, distribution, and reproduction in any medium, provided the original author(s) and source are credited.

\section{References}

1. European Committee for Veterinary Medicinal Products (1994) Chloramphenicol summary report. European Agency for the
Evaluation of Medicinal Products (EMEA). http://www.emea. europa.eu/pdfs/vet/mrls/chloramphenicol.pdg

2. Wongtavatchai J, McLean L.G, Ramos F, Arnold D (2004) WHO Food Additives series 53: Chloramphenicol. JECFA (WHO: Joint FAO/WHO Expert Committee on Food Additives), IPCS (International Programme on Chemical Safety) INCHEM, pp 7-85

3. Official Journal of the European Communities, L348/154-156 of 20 December 2002. Commission Decision 2002/994/EC Concerning certain protective measures with regard to the products of animal origin imported from China

4. Official Journal of the European Communities, L 260/35-36 of 27 September 2002. Commission Decision 2001/705/EC Concerning certain protective measures with regard to certain fishery and aquaculture products intended for human consumption and originating in Indonesia

5. Official Journal of the European Communities, L84/77-78 of 27 March 2002. Commission Decision 2002/251/EC Concerning certain protective measures with regard to poultry meat and certain fishery and aquaculture products intended for human consumption and imported from Thailand

6. Official Journal of the European Union; Commission Decision 2003/181/EC

7. Kanarat S, Tangsirisup N, Nijthavorn N, Elliott C, Cannavan A (2008) An investigation into the possible occurrence of chloramphenicol in poultry litter. In: van Ginkel LA, Bergwerff AA (eds) Proceedings from the EuroResidue IV Conference on residues of veterinary drugs in food, 19-21 May 2008, Egmond aan Zee, the Netherlands, vol. 1, ISBN/EAN: 978-90-804925-3-0

8. Boxall ABA, Johnson P, Smith EJ, Sinclair CJ, Stutt E, Levy LS (2006) Uptake of veterinary medicines from soils into plants. J Agric Food Chem 54:2288-2297

9. Kaufmann A, Butcher P (2005) Quantitative liquid chromatography/tandem mass spectrometry determination of chloramphenicol residues in food using sub-2 $\mu \mathrm{m}$ particulate high-performance liquid chromatography columns for sensitivity and speed. Rapid Com Mass Spectrom 19:3694-3700

10. Kennedy DG, McCracken RJ, Cannavan A, Hewitt SA (1998) Use of liquid chromatography-mass spectrometry in the analysis of residues of antibiotics in meat and milk. J Chromatogr A 812:77-98

11. Stolker AAM, Brinkman UATh (2005) Analytical strategies for residue analysis of veterinary drugs and growth-promoting agents in food-producing animals - a review. J Chromatogr A 1067:1553

12. Rønning HT, Einarsen K, Asp TN (2006) Determination of chloramphenicol residues in meat, seafood, egg, honey, milk, plasma and urine with liquid chromatography-tandem mass spectrometry, and the validation of the method based on 2002/ 657/EC. J Chromatogr A 1118:226-233

13. Schürmann A, Dvorak V, Crüzer C, Butcher P, Kaufmann A (2009) False-positive liquid chromatography/tandem mass spectrometric confirmation of sebuthylazine residues using the identification points system according to EU directive 2002/657/ EC due to a biogenic insecticide in tarragon. Rapid Com Mass Spectrom 23:1196-1200

14. Official Journal of the European Communities, L221/8-36 of 12 August 2002. Commission Decision 2002/657/EC implementing Council Directive 96/23/EC concerning the performance of analytical methods and the interpretation of results 